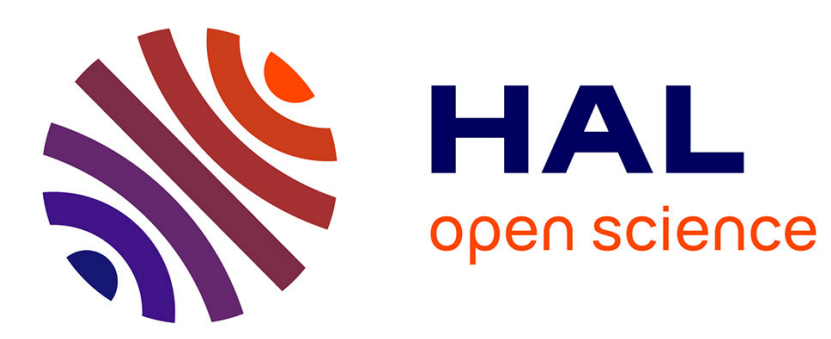

\title{
Improvement of signal to noise ratio in electro optical probing technique by wavelets filtering
} Anthony Boscaro, S Jacquir, K Sanchez, P Perdu, Stéphane Binczak

\section{To cite this version:}

Anthony Boscaro, S Jacquir, K Sanchez, P Perdu, Stéphane Binczak. Improvement of signal to noise ratio in electro optical probing technique by wavelets filtering. Microelectronics Reliability, 2015, Proceedings of the 26th European Symposium on Reliability of Electron Devices, Failure Physics and Analysis, 55 (9-10), pp.1585-1591. 10.1016/j.microrel.2015.06.100 . hal-01465725

\section{HAL Id: hal-01465725 \\ https://hal.science/hal-01465725}

Submitted on 13 Feb 2017

HAL is a multi-disciplinary open access archive for the deposit and dissemination of scientific research documents, whether they are published or not. The documents may come from teaching and research institutions in France or abroad, or from public or private research centers.
L'archive ouverte pluridisciplinaire HAL, est destinée au dépôt et à la diffusion de documents scientifiques de niveau recherche, publiés ou non, émanant des établissements d'enseignement et de recherche français ou étrangers, des laboratoires publics ou privés. 


\title{
Improvement of signal to noise ratio in Electro Optical Probing technique by wavelets filtering
}

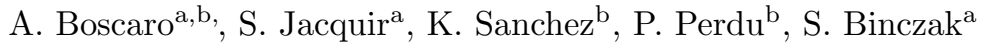 \\ ${ }^{a}$ Le2i, UMR CNRS 6306, Univ. Bourgogne Franche-Comté, 9 Avenue Alain Savary, 21000 Dijon, France \\ ${ }^{b}$ Centre National d'Etudes Spatiales (CNES), 18 Avenue Edouard Belin, 31401 Toulouse, France
}

\begin{abstract}
Electro Optical Probing (EOP) technique is an efficient backside contacless technique to measure waveforms in modern VLSI circuits. The signal related intensity variation of the reflected beam is very weak therefore, to acquire a signal with enough Signal to Noise Ratio, averaging techniques are usually performed. Resulting acquisition time for one waveform are too long to implement point to point probing to image mode. To overcome this limitation, we have developped a new filtering by wavelets approach to keep a good SNR while significantly reducing this acquisition time. It opens the doors to new multipoint probing applications. In this paper, we describe the technique, its efficiency in terms of SNR, execution time and limits.
\end{abstract}

\section{Corresponding author.}

Anthony Boscaro

Ph.D Student Laboraroire LE2i UMR CNRS 6306

UFR Sciences et Techniques

9 Avenue Alain Savary 21000 DIJON, France

Tel : +33 7.70.02.30.76

Email address : anthony.boscaro@u-bourgogne.fr 


\section{Introduction}

In addition to light emission techniques, methods based on laser exploit optical stimulation or optical properties of reflected beam [1]. Electro Optical Probing is a popular timing-analysis laser-based technique [2], using FranzKeldysh effects and free carrier absorption. Heinrich was the precursor of EOP approach [3] by proposing a noninvasive method which uses an infrared laser measuring the modulation of the free carrier density induced by the variation of electric potentials inside bipolar transistors. At the end of the nineties, a commercial application of the LVP technique was developped by Schlumberger Technologies Inc [4]. The source used was a pulsed laser, wavelength of 1.06 microns, 35 ps pulse duration.

Since its introduction, Electro Optical Probing has become an essential tool to the failure analysis (FA) and design debug communities [2]. In backside EOP analysis, pulsed or continuous laser beam is focused on a node of the chip which has been thinned. Thereafter, the reflected beam properties are analyzed.

The reflected signal mostly contains usual optical reflection from metal lines and a very weak signal related to free carrier absorption and bandgap modulation difficult to extract from noise. Techniques such as averaging [5] and frequency-domain measurements [6] have been implemented in probing and image modes. Averaging process is quite efficient in probing mode but it results at least in seconds duration to probe each node. This single waveform acquisition duration prevents EOP to probe each point of the device to build a waveform database of the full device. It will last from days to weeks to do it. Using frequency analysis in image mode is pretty fast but timing information is lost as a scan gives only amplitude information on one frequency inside the device. Therefore, in order to build a waveform database of the full device or just to reduce acquisition time to acquire thousands of nodes in a batch process (automatically moves to the next node, focus, slightly defocus up and down and each time scans a small area to finally extract the best waveform), it is mandatory to reduce processing time for each waveform while keeping a reasonable SNR. This paper introduces a new processing scheme based on wavelet transform to achieve this goal. We firstly remind some EOP background. The second section is dedicated to the description of EOP acquisition setup. The third section introduces the complete processing sequence. The fourth section exhibits the SNR improvement by wavelets transform. A fith section concerns examples of results. And before a conclusion a sixth section tells about the level of decomposition and the choice of the wavelet used for the filtering.

\section{Electro Optical probing}

EOP technique is still based on analysis of the reflected beam properties [7]. Intensity varies with temperature change [8], charge density [4] or electric field [9]. The analyze of the properties of the reflected laser beam can be traced back to its origin, in order to obtain information on the physical parameter studied. Two wavelengths have been used for EOP, also known as LVP (Laser Voltage Probing, [10] [11]) : 1064 and 1340 nm. 1064 gives a better spatial resolution (shorter wavelength) and the laser beam absorption is sensitive to carrier density (free carrier absorption) and to small bandgap (Franz Keldish) variations. Nevertheless, absorption is important (less reflected beam intensity) and it induces slight Optical Beam Induced Current (OBIC) that could bias a little bit the measurements. $1340 \mathrm{~nm}$ is less absorbed, is not sensitive to Franz Keldish effect. Considering the free carrier absorption mechanism, EOP is based on the very weak useful part of the reflected signal. It means that noise coming from various sources (thermal, shotky, electronic ...) is often order of magnitude higher than this useful part of the signal. During the acquisition, a signal with a weak amplitude drowned in noise is measured. If the waveform is directly acquired without processing, the SNR is extremely low. The absorption coefficient varies according to equation (1) [12]. According to M. Rebai [12], the relative changes are often less than $10^{-3}$ less than those induced by temperature changes.

$$
\alpha_{f c}=A \frac{\lambda^{2} q^{3}}{4 \pi^{2} c_{0}^{3} \epsilon_{0} n_{0}}\left[\frac{N_{e}}{m_{e}^{2} \mu_{e}}+\frac{N_{h}}{m_{h}^{2} \mu_{h}}\right]
$$

with $\alpha_{f_{c}}=$ absorption coefficient variation, $\mathrm{A}=$ refinement coefficient, $\lambda$ = wavelength, $\mathrm{q}=$ elementary charge, $c_{0}=$ celerity of light in vacuum, $\epsilon_{0}=$ permittivity in vacuum, $n_{0}=$ refractive index in silicon, $\left[m_{e}, m_{h}\right]=$ concentration of electrons and holes, $\left[\mu_{e}, \mu_{h}\right]=$ mobility of electrons and holes, $\left[N_{e}, N_{h}\right]=$ number of electrons and holes.

\section{Acquisition setup}

To solve this problem and improve the SNR, our existing aquisition setup integrates signals by averaging. Experimentations were done on Tri-Phemos, device designed by HAMAMATSU Photonics.

The signal of the reflected beam is acquired by a photodiode and amplified. The output signal of the amplifier is sent to an oscilloscope which proceeds in 512 averages to improve the SNR in a first time. In a second time, this averaged signal is sent to a computer (with LABVIEW interface) which integrates by averaging severals acquisitions (one acquisition corresponds to 512 oscilloscope averages). Figure 1a shows that the signal is submerged in noise if only one acquisition is taken into account. Our setup needs several averages, by consequences a lot of time is necessary to significantly improve SNR. To illustrate these explanations, an example of probing on a micocontroler STM32 was chosen. After some averages (10 averages in our case), we obtain the waveform in FIGURE 1b. We can notice that 


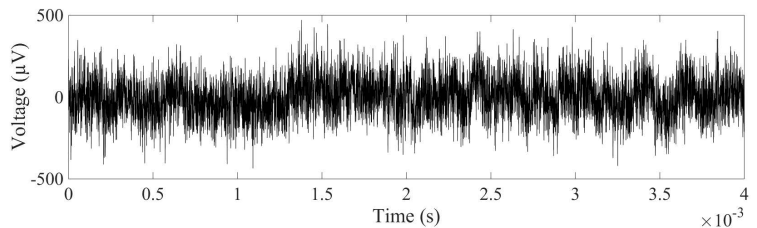

(a)

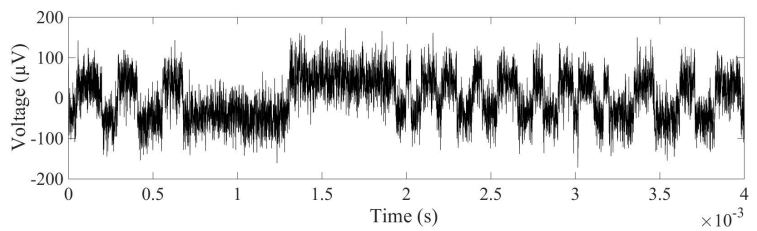

(b)

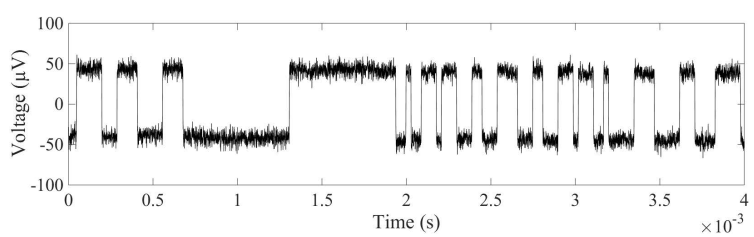

(c)

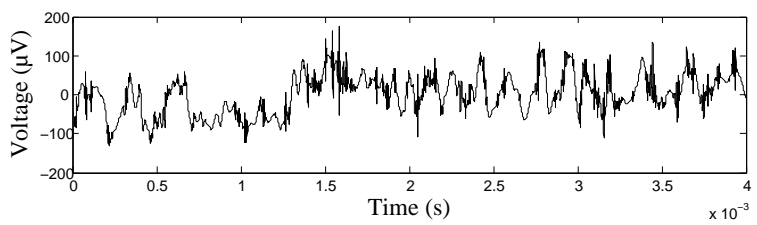

(d)

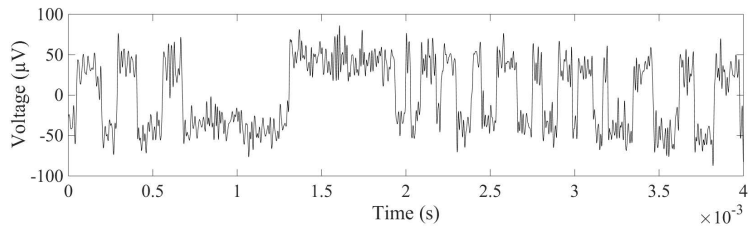

(e)

Figure 1: Probing on Digital/Analog converter STM32: (a) One acquisition of EOP signal / Computing Time : $\approx 0.7 \mathrm{~s} / \mathrm{SNR}=$ $-19.96 \mathrm{~dB}$. (b) Signal rebuild with 10 averages / Computing Time : $\approx 7 \mathrm{~s} / \mathrm{SNR}=3.09 \mathrm{~dB}$. (c) Signal rebuild with 418 averages / Computing Time $: \approx 292 \mathrm{~s} / \mathrm{SNR}=37.39 \mathrm{~dB}$. (d) Signal rebuild with wavelets transform and 1 average/Computing Time $: \approx 0.8 \mathrm{~s}$ $/ \mathrm{SNR}=1.9 \mathrm{~dB}$. (e) Signal rebuild with wavelets transform and 10 averages / Computing Time $: \approx 8 s / \mathrm{SNR}=22.9 \mathrm{~dB}$.

the SNR is very low. The number of averages has been increased to decrease the SNR. FIGURE 1c is the result after 418 averages.

Considering our hardware, an acquition is achieved in $0.7 \mathrm{~s}$. It is very long in some cases as shown with the previous example. An approach with simple filters (Median, FIR, IIR) has been tested to improve the SNR and reduce acquisition time. If this kind of process is applied with a large scale, the signal will be indeed smoothed but short duration signals can be lost because of mandatory sample size (up to tens of samples) to get a reasonable noise re- duction. That is why a process based on filtering by wavelets was implemented to improve both aquisition times and SNR. Example of result is represented in FIgURE 1. We precise that the aim of this process is to decrease the number of averages in order to reduce aquisition time while having a good SNR. For this example we have taken the same signal as previously but only 10 averages are used. Visually, we can notice the improvement of the SNR. In addition the acquition time is reduced due to the decreasing of averages. For the same acquisition time ( $7 \mathrm{~s}$ here), the SNR is significantly improved. The computing time for wavelet transform can be evaluated in few $\mathrm{ms}$. The filtering process by wavelets transform is explained below.

\section{SNR improvement by wavelets transform}

\subsection{Multiresolution analysis : discrete wavelet transform}

Since few years, several approaches have emerged in signal processing in order to remove the noise in signals. In [13], it is said that these new methods with wavelets give better results than Wiener filtering. Wavelets transform can be adapted to the discrete set. The size of the information could be reduced by choosing a level of detail. In our case, we use one aspect of wavelets : the multiresolution decomposition, which is very used in image processing because of its performance [14]. It is recalled that in failure analysis, wavelets have ever been applied to perform the approach by Time-Resolved Imaging in light emission [15]. This reference used the Continous Wavelet Transform to determine a frequency while we use the discrete wavelet transform (DWT) to filter the signal. [16] and [17] explain the theory of the DWT. Given a signal $s$ of length $N$, the DWT consists of $\log _{2} N$ stages at most. The first step produces, starting from $s$, two sets of coefficients : approximation coefficients $C A_{i}$, and details coefficients $C D_{i}$, with $\mathrm{i}=[1, \ldots, \mathrm{n}]$. These vectors are obtained by convoluting $s$ with an expression equivalent to a low-pass filter $\left(l_{d}\right)$ for approximation, and with another equivalent to an highpass filter $\left(h_{d}\right)$ for details, followed by under-sampling [16]. $\left(l_{d}\right)$ and $\left(h_{d}\right)$ correspond exactly with impulse response of low and high-pass filter respectively. These coefficients are described such as

$$
\begin{aligned}
& C D_{i}[n]=\sum_{k=-\infty}^{\infty} h_{d}[k] s[2 n-k], \\
& C A_{i}[n]=\sum_{k=-\infty}^{\infty} l_{d}[k] s[2 n-k],
\end{aligned}
$$

where $\mathrm{n}$ and $\mathrm{k}$ denote discrete time coefficient, and $i \in \mathbb{Z}$. In this way, the signal $s$ can be written as

$$
s[n]=\sum_{k=-\infty}^{\infty}\left(C D_{i}[k] h_{r}[2 k-n]+C A_{i}[k] l_{r}[2 k-n]\right),
$$

where $l_{r}$ and $h_{r}$ are reconstitution filters. Next step splits the approximation coefficient $C A_{1}$ in two parts using the 


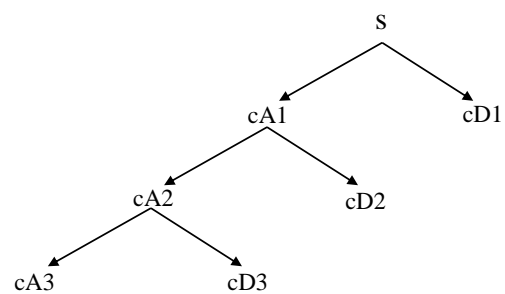

Figure 2: Multiresolution decomposition tree for level $L=3$.

same scheme, replacing $s$ by $C A_{1}$, and producing $C A_{2}$ and $C D_{2}$, and so on. The wavelet decomposition at level $L$ has the following struture : $\left[C A_{i}, C D_{i}, \ldots, C D_{1}\right]$. Figure 2, represents this struture with terminal nodes.

Now the important step, is to understand how to denoise the signal $\boldsymbol{S}$. For that purpose, an approach by coefficients thresholding was chosen [18] and described in the next section.

\subsection{Coefficients thresholding}

According to the literature, it exists different kinds of thresholding for the wavelets coefficients [13] :

- Soft thresholding : The absolute value of all the wavelets coefficients is compared to a threshold $\mathrm{T}$. If this value is greater than $\mathrm{T}$ the threshold is subtracted from any coefficient that is greater than the threshold. Others are set to zero.

- Hard thresholding : Hard thresholding sets any coefficient less than or equal to the threshold $\mathrm{T}$ to zero. Others are preserved.

- Universal thresholding : the value called universal, is defined by :

$$
T_{\text {thresh }}=\sigma \sqrt{2 \log (N)}
$$

where $\mathrm{N}$ is the length of the signal and $\sigma$ the noise's standard deviation. Here, $T_{\text {thresh }}$ is used with hard thresholding. In several applications, noise is most of the time white and gaussian. This kind of white guaussian noise (WGN) is a random signal with constant power spectral density. WGN whose representation is given by (6), is independant and identically distributed (i.i.d) and drawn from a zero-mean normal distribution with variance $\sigma^{2}$.

$$
W G N \sim N\left(0, \sigma^{2}\right)
$$

According to (6), only $\sigma^{2}$ is unknown. That is why wavelets are useful in our study. Here, only the standart deviation could be estimated. In rarely cases the noise is assumed but in others, it can be estimated by using the Median Absolute Deviation (MAD). This method has been introduced by Donoho and Johnstone in 1994 [19]. MAD is the median absolute deviation of the empirical wavelet coefficients corresponding to the highest level $j 1$. The reason for using these highest level coefficients for the variance estimation, is that they are mostly costitued of noise [20]. By consequences the estimated variance is given by

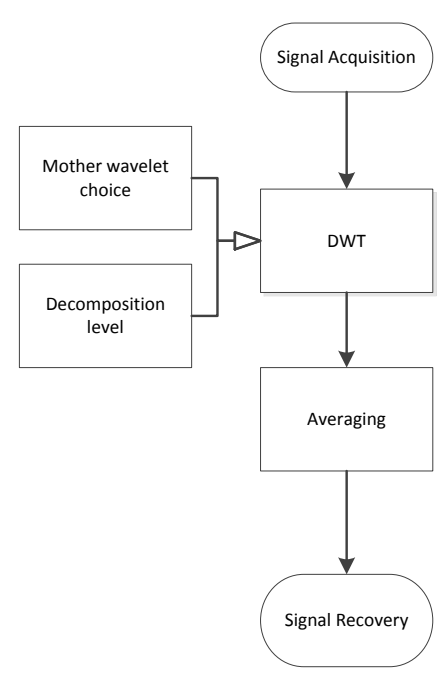

FIGURE 3: Flowchart of the filtering by wavelets.

$$
\sigma^{2}=\left(\frac{M A D}{0.6745}\right)^{2}
$$

where 0.6745 is the 0.75 - quantile of the standard normal distribution and

$$
M A D\left(\left(w_{j}\right)\right)=\operatorname{Median}\left(\left(\left|w_{j}\right|\right)_{j}\right)
$$

with $w_{j}$, the wavelets coefficients. Thus, $\sigma^{2}$ is now known and the threshold given in (5) can be computed. For a better understanding, a flowchart of our new process is illustrated in FiguRE 3.

\section{Application, results and discussion}

\subsection{Results on STM32 microcontroller}

The process is applied on digital signal acquired on a $90 \mathrm{~nm}$ microcontroller STM32, more precisly on the digital analog converter. The topographic image is reported in Figure 4. On this picture, we can notice a yellow cross which represents the pointer where the probing is applied. FIGURE 5 illustrates the acquired waveform and its wavelets coefficients decomposition. For this example we have decomposed with four levels with an Haar mother wavelet. On this picture we start by decomposing the original signal with wavelets. The next step consists to apply a threshold for the coefficients. And finally we go back to the time domain. As you can see at the right in Figure 5 , we can considerably denoise the signal.

\subsection{Signal to Noise Ratio}

Previously, it has been seen that it was possible to denoise the signal. It could be interesting to compare this new method with available filtering method in the LVP field. Our filtering by wavelets is compared to filtering by 


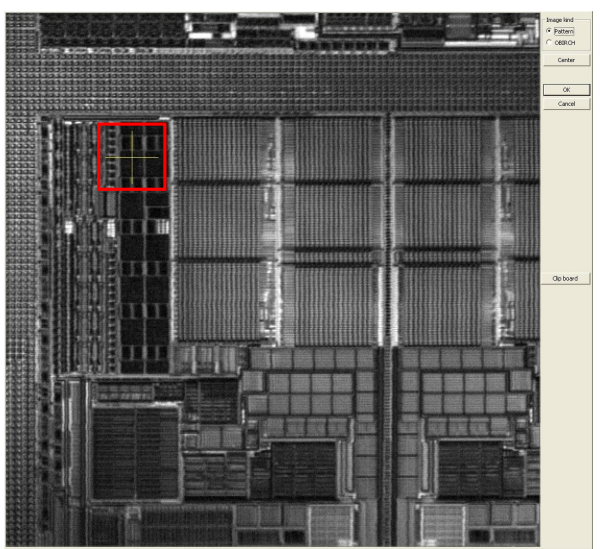

Figure 4: Pattern of microcontroller Digital/Analog converter (DAC) STM 32 and probing area symbolized by the yellow cross.
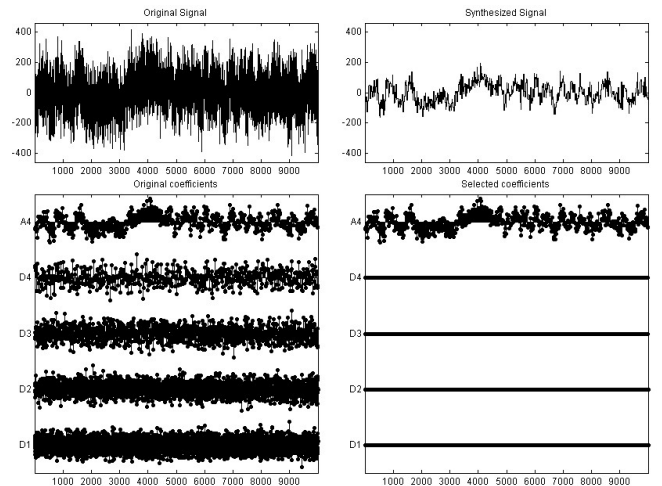

Figure 5: Wavelets decomposition with four levels. Top left : Noised signal/ Bottom left : Wavelets coefficients/Top right : Denoised signal/Bottom right : Thresholded coefficients.

averaging and median filter. FiguRE 6 represents the evolution of the SNR in function of the number of averages for each case. Here SNR is given by the following equation :

$$
S N R_{(d B)}=10 \log _{10} \frac{\operatorname{Var}(\text { Signal })}{\operatorname{Var}(\text { Noise })}
$$

where $\operatorname{Var}$ (Signal) and $\operatorname{Var}($ Noise) correspond to the signal and noise variance respectively.

For each values of average, the SNR with filtering by wavelets is always higher than the SNR computed with average and median filter. It proves the efficiency of the filtering to reconstitute the signal with a good SNR. Now we will focus on the other key aspect of this study, more precisly the execution time and the reconstitution error (mean square error).

\subsection{Acquisition time}

After the examination of the SNR, we give some results concerning the acquition time which is not a negligable parameter. In fact, by decreasing the number of averages, the acquisition time decreases. Different acquisitions have

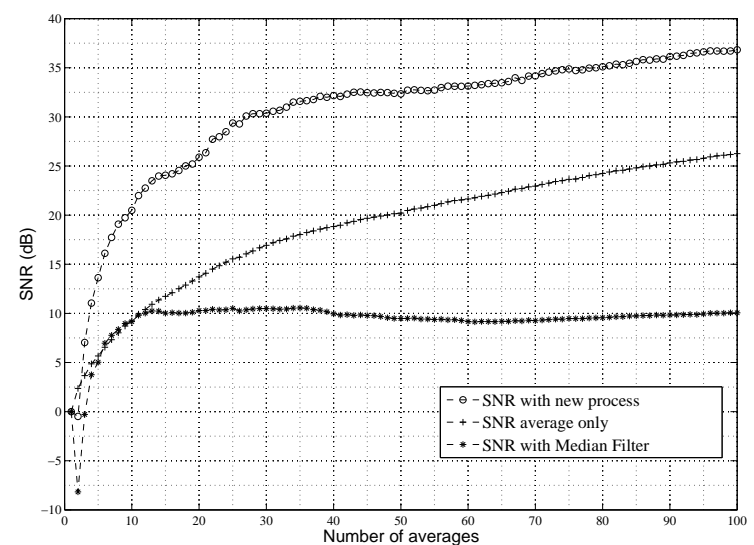

FIGURE 6: SNR evolution with wavelets, average and median filtering.

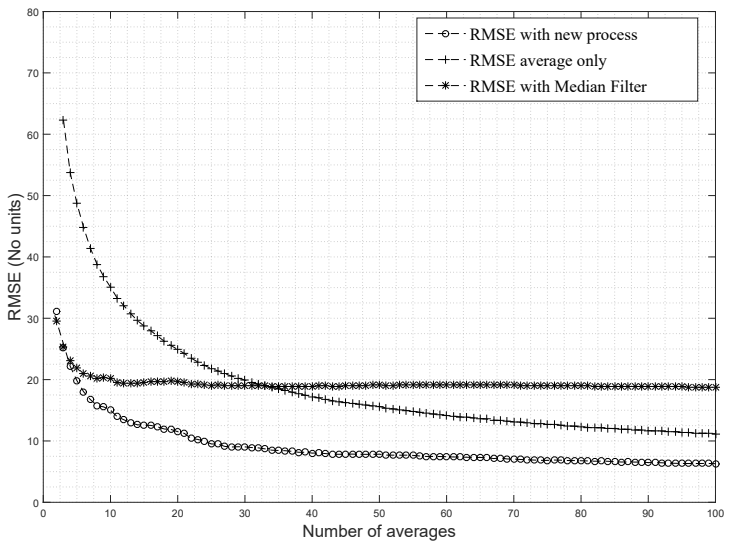

FiguRE 7: Mean Square Error evolution with new filtering process, averaging and median filtering.

been realized on some analogue or digital signals. For a same SNR, we have reported the execution time for all the signals. Results are refered in TABLE 1. In all cases, the execution time with only average and median filter is always higher than the computing time with wavelets. We notice a gain between 10 and $30 \mathrm{~dB}$, depending on cases.

\subsection{Reconstitution error : Mean Square Error}

In this king of study, it is interresting to compare quantitatively each method. That is reason the Mean Square Error (MSE) is used here. If $\hat{x}$ is a vector of $\mathrm{n}$ predictions, and $x$ is the vector of the true values, then the MSE of the predictor is given by :

$$
M S E=\frac{1}{n} \sum_{i=1}^{n}(\hat{x}-x)^{2}
$$

Thus, the MSE evolution is computed for each kind of filter, average, median and wavelets and is represented in FIgURE 7 . We can notice immediately that the MSE is minimized in the case of wavelets filtering for each number of 
TABLE 1: Results of execution time on seven signals (analogue/digital) with wavelets and averaging, with median filter and with averaging only.

\begin{tabular}{|c|c|c|c|c|c|c|c|}
\hline SNR $=30 \mathrm{db}$ & Signal 1 & Signal 2 & Signal 3 & Signal 4 & Signal 5 & Signal 6 & Signal 7 \\
\hline Number of acquisitions with wavelets & $\mathbf{5}$ & $\mathbf{1 5}$ & $\mathbf{1 0}$ & $\mathbf{2 0}$ & $\mathbf{1 4}$ & $\mathbf{2 5}$ & $\mathbf{6}$ \\
\hline Time with wavelets and averaging (s) & 3.5 & 10.5 & 7 & 14 & 9.8 & 17.5 & 4.2 \\
\hline Number of acquisitions with averaging & 20 & 70 & 50 & 100 & 40 & 120 & 25 \\
\hline Time with averaging (s) & 14 & 49 & 35 & 70 & 28 & 84 & 17.5 \\
\hline Number of acquisitions with median filter & 10 & 35 & 34 & 60 & 22 & 80 & 17 \\
\hline Time with median filter (s) & 8 & 25 & 19 & 42 & 15 & 56 & 12 \\
\hline
\end{tabular}

TABLE 2: Mean Square Error (MSE) in function of the mother wavelet choice.

\begin{tabular}{|c|c|c|c|c|c|c|c|c|c|c|c|}
\hline \multicolumn{2}{|c|}{ Wavelet } & \multicolumn{10}{|l|}{ MSE } \\
\hline & & Sig 1 & $\begin{array}{l}\text { Sig } 2 \\
\end{array}$ & Sig 3 & Sig 4 & Sig 5 & Sig 6 & Sig 7 & Sig 8 & $\begin{array}{l}\text { Sig } 9 \\
\end{array}$ & Sig 10 \\
\hline 1 & Sym 2 & 0.0163 & 0.0166 & 0.0143 & 0.0157 & 0.0153 & 0.0148 & 0.0157 & 0.0164 & 0.0131 & 0.0139 \\
\hline 2 & Sym 4 & 0.0144 & 0.0149 & 0.0132 & 0.0144 & 0.0149 & 0.0142 & 0.0152 & 0.0161 & 0.0129 & 0.0133 \\
\hline 3 & Sym 8 & 0.0155 & 0.0152 & 0.0143 & 0.0167 & 0.0157 & 0.0138 & 0.0148 & 0.0168 & 0.0123 & 0.0128 \\
\hline 4 & Sym 16 & 0.0142 & 0.0145 & 0.0131 & 0.0154 & 0.0144 & 0.0153 & 0.0149 & 0.0157 & 0.0117 & 0.0129 \\
\hline 5 & Sym 32 & 0.0152 & 0.0153 & 0.0147 & 0.0153 & 0.0162 & 0.0149 & 0.0162 & 0.0159 & 0.0135 & 0.0134 \\
\hline 6 & Db 2 & 0.0151 & 0.0147 & 0.0152 & 0.0164 & 0.0156 & 0.0154 & 0.0153 & 0.0165 & 0.0141 & 0.0145 \\
\hline 7 & $\mathrm{Db} 4$ & 0.0162 & 0.0155 & 0.0158 & 0.0163 & 0.0155 & 0.0168 & 0.0172 & 0.0176 & 0.0152 & 0.0148 \\
\hline 8 & Db 8 & 0.0161 & 0.0164 & 0.0167 & 0.0171 & 0.0166 & 0.0176 & 0.0175 & 0.0181 & 0.0164 & 0.0156 \\
\hline 9 & Db 16 & 0.0182 & 0.0178 & 0.0169 & 0.0159 & 0.0184 & 0.0194 & 0.0154 & 0.0189 & 0.0172 & 0.0157 \\
\hline 10 & Db 32 & 0.0193 & 0.0187 & 0.0184 & 0.0198 & 0.0193 & 0.0197 & 0.0156 & 0.0194 & 0.0184 & 0.0161 \\
\hline 11 & Coif 1 & 0.0161 & 0.0165 & 0.0162 & 0.0169 & 0.0158 & 0.0168 & 0.0171 & 0.0178 & 0.0154 & 0.0166 \\
\hline 12 & Coif 2 & 0.0144 & 0.0152 & 0.0154 & 0.0157 & 0.0152 & 0.0155 & 0.0169 & 0.0159 & 0.0147 & 0.0163 \\
\hline 13 & Coif 3 & 0.0155 & 0.0153 & 0.0162 & 0.0162 & 0.0164 & 0.0167 & 0.0173 & 0.0175 & 0.0149 & 0.0159 \\
\hline 14 & Coif 4 & 0.0145 & 0.0141 & 0.0144 & 0.0165 & 0.0150 & 0.0153 & 0.0156 & 0.0163 & 0.0137 & 0.0154 \\
\hline 15 & Coif 5 & 0.0152 & 0.0159 & 0.0157 & 0.0164 & 0.0163 & 0.0166 & 0.0169 & 0.0168 & 0.0142 & 0.0156 \\
\hline 16 & Haar & 0.0198 & 0.0196 & 0.0214 & 0.0189 & 0.0221 & 0.0217 & 0.0198 & 0.0243 & 0.0197 & 0.0211 \\
\hline 17 & Meyer & 0.0151 & 0.0153 & 0.0164 & 0.0158 & 0.0168 & 0.0174 & 0.0175 & 0.0172 & 0.0156 & 0.0158 \\
\hline
\end{tabular}

average. The quantitative analysis with SNR and execution time is reinforced by the MSE to prove the efficency of our process.

\subsection{Other Example}

To illustrate graphically our results, FIGURE 8 represents a comparison between the different kinds of filters used in LVP. This example has been realized on digital signal acquired on a $90 \mathrm{~nm}$ microcontroller STM32. In a first time, one acquisition is realized, see FIGURE 8(a), and then wavelets filtering is applied on 5 averaging, see Figure 8(b). Five averaging are reprensented in Figure 8 (c) and finally median filter is applied on 5 averaging FIGURE 8(d) in order have a comparison. Each signal has been averaged five times in order to reduce the computing time. For a same number of averages, the signal in FiguRE 8(b) has clearly the best SNR.

\section{Discussion}

\subsection{Decomposition Level}

In this paper, prior parameters must be chosen, more precisly the decomposition level $(D L)$ and the mother wa- velet $(M W)$. In fact the aim is to automate the filtering process. Concerning the decomposition level, it is possible to compute it automaticaly.

In the wavelets theory the DL max is given by the following equation :

$$
D L_{\max }=\log _{2} N
$$

with $\mathrm{N}$ the signal length. Thus, if the signal length is known, $D L_{\max }$ can be computed easily.

\subsection{Mother wavelet}

Selection of mother wavelet $(M W)$ could be based on qualitative or quantitative approaches. The chosen database are ten signals (analogue and digital). In this study, different MW with different vanishing moments were used : Symmlet(Sym), Daubechises(Db), Haar, Meyer and Coiflet (Coif). Instead of using qualitive approach such as similarity between signal and MW, this discussion will be based on the MSE value in function of the MW, i.e. the MSE is computed for each mother wavelet and for each vanishing moment. In fact for transcient signals, using Haar wavelet could be pointed because its shape is similar to 


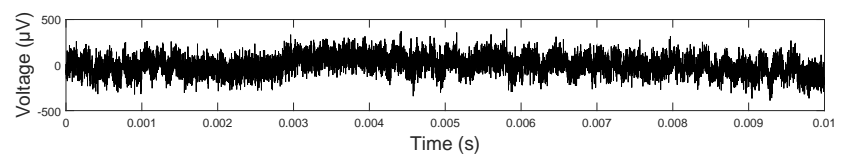

(a)

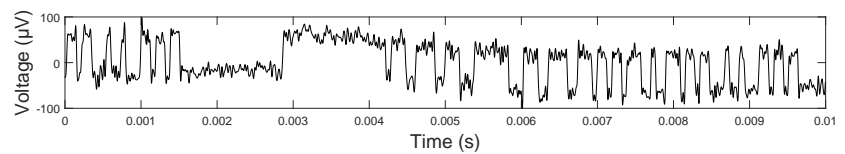

(b)

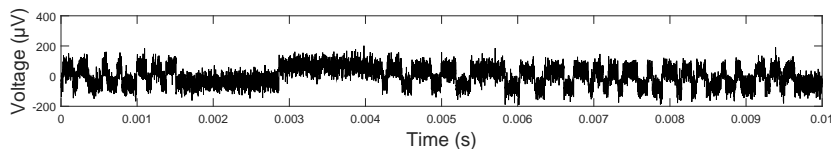

(c)

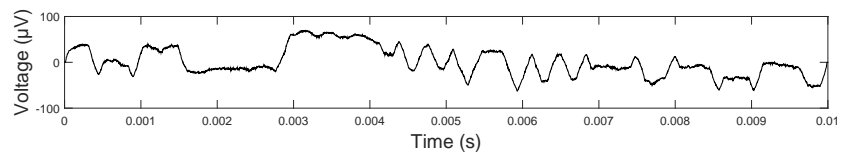

(d)

Figure 8: (a) Signal with one acquisition. (b) Signal with wavelets filtering (c) Signal with averaging filter (d) Signal with median filtering (size 50)

numeric signal. But it does not prove that it is the good choice, that is the reason a quantitative approach has been chosen. Results are liste in TABLE 2 . In $80 \%$ of cases the Sym 16 mother wavelet gives the best results. These results give credit to the idea of choosing a mother wavelet to minimize the MSE.

\section{Conclusion and perspectives}

Rebuild a weak signal is a real challenge especialy when it is drowned in noise. In this paper, a new method based on compression has been reported. This process could be useful for the FA community because its allows the expert to significaly save time during the acquisition. Therefore it is partially automated, in fact the mother wavelet choice is a key step of the process and the most difficult. If the MW is chosen at the begining, the process is completly automated. In the event of the expert is not satisfied with result, he can manually adjust all the parameters to optimize the final SNR.

In terms of perspectives, this process opens the door to new multipoint probing applications and allows FA community to use signal processing techniques instead of expensive hardware.

\section{Acknowledgement}

Authors would like to thank Hamamatsu Photonics for its technical support (TriPHEMOS) and the council of Burgundy for its financial support.

\section{Bibliography}

[1] Chin Jiann Min, Narang Vinod, Zhao Xiaole, Tay Meng Yeow, Phoa Angeline,Ravikumar Venkat, Ei Lwin Hnin, Lim Soon Huat, Teo Chea Wei, Zulkifli Syahirah and others, "Fault isolation in semiconductor product, process, physical and package failure analysis : Importance and overview", Microelectronics Reliability ,vol. 51, pp. 1440-1448, 2011.

[2] Kindereit U, "Fundamentals and Future Applications of Laser Voltage Probing", IEEE-IRPS , pp. 162-172, 2014.

[3] Heinrich HK, "Picosecond noninvasive optical detection of internal electrical signals in flip-chip-mounted silicon integrated circuits", IBM journal of research and development , pp. 162-172, 1990.

4] Paniccia M, Rao RM and Yee Wai Mun, "Optical probing of flip chip packaged microprocessors", Journal of Vacuum Science 8 Technology B , pp. 162-172, 1990.

[5] Wilsher Kenneth R, Palo Alto, Lo William K and Rajan Suresh N, "Dual-laser voltage probing of IC's", European patent application : EP 0864872 A2, 1998.

[6] Kindereit U, Woods G, Tian J, Kerst U and Boit C, "Investigation of laser voltage probing signals in CMOS transistors", Reliability physics symposium, 200\%. proceedings. 45 th annual. ieee international , pp. 526-633, 2007.

[7] Harley K Heinrich and David D Bloom, "Method and means for optical detection of charge density modulation in a semiconductor", American patent application : US 4758092 A, 1986.

[8] Quintard V, "Réflectométrie et interférométrie laser haute résolution. Application à la caractérisation de composants électroniques", PhD thesis.

[9] Heinrich HK, Hemenway BR, McGroddy KA and Bloom DM, "Measurement of real-time digital signals in a silicon bipolar junction transistor using a noninvasive optical probe", Electronics letters , pp. 650-652, 1986.

[10] Kolachina S, "Introduction to Laser Voltage Probing (LVP) of Integrated Circuits", SMicroelectronics Failure Analysis , pp. 349-353, 2011.

[11] A.E.SOREF and B.BRIAN, "Electro-optical Effects in Silicones", IEEE, vol. QE-23, pp. 123, 1987.

[12] Rebai MM and al, "Temperature Effect on Reflected Laser Probing Signal of Multiple Elementary Substructures", Proceedinds of the IEEE 21st International Symposium on the Physical and Failure Analysis of Integrated Circuits (IPFA), pp. 370-374, 2014.

[13] Walker and James S, "Tree-adapted wavelet shrinkage", Advances in Imaging and Electron Physics, vol. 124, pp. 343-394, 2002.

[14] Grgic Sonja, Grgic Mislav and Zovko-Cihlar Branka, "Performance analysis of image compression using wavelets", Industrial Electronics, IEEE Transactions on, vol. 48, pp. 682-695, 2001.

[15] Chef S, Jacquir S, Sanchez K, Perdu P and Binczak S, 'Frequency mapping in dynamic light emission with wavelet transform", Microelectronics Reliability, vol. 53, pp. 1387-1392, 2013.

[16] Mallat Stephane G, "A theory for multiresolution signal decomposition : the wavelet representation", Pattern Analysis and $M a-$ chine Intelligence, IEEE Transactions on, vol. 11, pp. 674-693, 1989.

[17] Meyer Y, "Wavelets and operators", Pattern Analysis and $\mathrm{Ma}$ chine Intelligence, IEEE Transactions on, vol. 11, pp. 674-693, 1989.

[18] Walczak B and Massart DL, "Noise suppression and signal compression using the wavelet packet transform", Cambridge university press, vol. 1, 1995.

[19] D.L. Donoho and J.M. Johnstone, "Ideal spatial adaptation by wavelet shrinkage", Biometrika, vol. 81, pp. 425-455, 1994.

[20] W. Härdle, G. Kerkyacharian, D. Picard and A. Tsybakov, "Wavelet thresholding and adapation", Wavelet, Approximation, and Statistical Application,pp. 193-213, 1998. 